\title{
RATIONALITY OF HILBERT SERIES IN NONCOMMUTATIVE INVARIANT THEORY
}

\author{
MÁTYÁS DOMOKOS AND VESSELIN DRENSKY
}

\begin{abstract}
It is a fundamental result in commutative algebra and invariant theory that a finitely generated graded module over a commutative finitely generated graded algebra has a rational Hilbert series, and consequently the Hilbert series of the algebra of polynomial invariants of a group of linear transformations is rational, whenever this algebra is finitely generated. This basic principle is applied here to prove rationality of Hilbert series of algebras of invariants that are neither commutative nor finitely generated. Our main focus is on linear groups acting on certain factor algebras of the tensor algebra that arise naturally in the theory of polynomial identities.
\end{abstract}

\section{INTRODUCTION}

A natural possible framework to develop noncommutative invariant theory is to investigate the action of a linear transformation group $G \leq G L(V)$ on the factor of the tensor algebra $K\langle V\rangle$ modulo a $G L(V)$-stable ideal $I$, where $V$ is a finite dimensional vector space over a base field $K$ of characteristic 0 . We shall assume that $\operatorname{dim}(V) \geq 2$. A special class of $G L(V)$-stable ideals in $K\langle V\rangle$ are the T-ideals. Recall that an ideal in $K\langle V\rangle$ is a $T$-ideal if it is stable with respect to all $K$-algebra endomorphisms of $K\langle V\rangle$. T-ideals are associated with varieties of associative $K$ algebras. The variety $\mathfrak{R}$ defined by a system of elements $J=\left\{f_{j}\right\}$ in the free associative algebra $K\left\langle x_{1}, x_{2}, \ldots\right\rangle$ of countable rank consists of all associative $K$ algebras $A$ such that $f_{j}=0$ is a polynomial identity on $A$ for all $f_{j} \in J$. Identifying the tensor algebra $K\langle V\rangle$ with the free associative algebra $K\left\langle x_{1}, \ldots, x_{n}\right\rangle$ of rank $n=\operatorname{dim}(V)$, the T-ideal $I=I_{n}(\mathfrak{R})$ is recovered as the set of $n$-variable identities satisfied by all algebras in a suitable variety $\mathfrak{R}$. Therefore in this case we shall write $K\langle V\rangle / I=F_{n}(\Re)$, and call it a relatively free algebra, since it is a free object generated by $n$ elements in the variety $\mathfrak{R}$. For a background on T-ideals and polynomial identities of associative algebras see [13].

We shall assume that $I_{n}(\mathfrak{R})$ is non-zero, so $F_{n}(\mathfrak{R})$ is not the free algebra. In the special case when $\mathfrak{R}$ is the variety of commutative algebras, we recover $S(V)=$ $K\left[x_{1}, \ldots, x_{n}\right]$, the symmetric tensor algebra of $V$, which can be thought of as the algebra of polynomial functions on the dual space $V^{*}$ of $V$. For surveys on the

2010 Mathematics Subject Classification. 16R10, 13A50, 13A02, 15A72, 16W22, 16W50, $05 \mathrm{E} 10$.

Key words and phrases. rational Hilbert series, Schur function, relatively free associative algebras, rational representation, unipotent subgroup, reductive group, tensor algebra, noncommutative invariant theory.

This project was carried out in the framework of the exchange program between the Hungarian and Bulgarian Academies of Sciences. It was partially supported by the Hungarian National Research, Development and Innovation Office, NKFIH K 119934 and by Grant I02/18 of the Bulgarian National Science Fund. 
study of $F_{n}(\Re)^{G}$ see [17] and [12. Loosely speaking, the moral of these works is that one can expect results analogous to the commutative case only when $F_{n}(\mathfrak{R})$ is not very far from the commutative polynomial algebra $K\left[x_{1}, \ldots, x_{n}\right]$. For example, benchmark results of commutative invariant theory are the statements that the algebras of (commutative) polynomial invariants of finite groups or more generally, reductive groups are finitely generated. Kharchenko 22] characterized the class of varieties $\mathfrak{R}$ such that $F_{n}(\mathfrak{R})^{G}$ is finitely generated for all finite groups $G$, and this class turned out to be rather narrow. The present authors in [11] characterized the even narrower class of varieties such that $F_{n}(\mathfrak{R})^{G}$ is finitely generated for all reductive $G$. Furthermore, we mention that the first named author in 9 ] extended the Sheppard-Todd-Chevalley Theorem by showing that $F_{n}(\mathfrak{R})^{G} \cong F_{n}(\mathfrak{R})$ holds for some finite group $G$ if and only if $G$ is a pseudoreflection group and the T-ideal $I(\Re)$ contains $\left[\left[x_{1}, x_{2}\right], x_{2}\right]$, where we write $[a, b]=a b-b a$.

In contrast with that, in the present paper we deal with a general fact from commutative invariant theory that remains valid for all noncommutative relatively free algebras. Namely, by the so-called Hilbert-Serre Theorem, whenever $K\left[x_{1}, \ldots, x_{n}\right]^{G}$ is finitely generated, its Hilbert series is rational. Notably this holds when $G$ is reductive or $G$ is a maximal unipotent subgroup of a reductive subgroup of $G L(V)$. Our main result Theorem 5.1 is that the Hilbert series of the graded algebra $F_{n}(\mathfrak{R})^{G}$ is rational, provided that $G \leq G L(V)$ is a group for which the finite generation property holds for subalgebras of $G$-invariants in finitely generated commutative graded algebras $R$ on which $G L(V)$ acts rationally (in particular, for a closed reductive subgroup $G$ of $G L(V)$ or for a maximal unipotent subgroup $G$ of a closed reductive subgroup of $G L(V))$. Consequently, although $F_{n}(\mathfrak{R})^{G}$ is rarely finitely generated even for reductive groups, it always has rational Hilbert series for $G$ reductive or a maximal unipotent subgroup of a reductive subgroup of $G L_{n}$.

A key ingredient of our proof is that extending a result of Belov [4, Berele [5] proved that the multivariate Hilbert series of a relatively free algebra is of special form (called nice by him). In Section 2 (working in a more general setup than [5]), we give in Proposition 2.2 a characterization of nice rational symmetric functions in terms of formal characters of the general linear group $G L_{n}$ and finitely generated modules over commutative polynomial algebras, endowed with a compatible rational $G L_{n}$-action. This characterization allows us to deduce in Section 3 the general Theorem 3.4 about rationality of the Hilbert series of the subspace of $G$-invariants in a graded vector space on which $G L_{n}$ acts rationally such that the formal character is a nice rational function, provided that the subgroup $G \leq G L_{n}$ satisfies a condition formulated in terms of commutative invariant theory. In Section 4 we discuss the problem for the rationality of the Hilbert series of non-finitely generated algebras of commutative polynomial invariants. We compute the Hilbert series of a textbook variant of the famous example of Nagata of an algebra of commutative invariants which is not finitely generated. It turns out that this algebra still has a nice rational Hilbert series. We also give an example of a normal graded subalgebra of $K\left[x_{1}, x_{2}\right]$ with transcendental Hilbert series, and formulate Problem 4.3 concerning rationality of Hilbert series in commutative invariant theory. In Section 5 we apply Theorem 3.4 for relatively free algebras, and deduce by the above mentioned result of Belov and Berele our main result Theorem 5.1 on rationality of the Hilbert series of the subalgebra of invariants $F_{n}(\Re)^{G}$ for a notable class of groups $G \leq G L_{n}$. In Section 6 we give examples which show that the condition that the multivariate 
Hilbert series of the relatively free algebra is a nice rational function cannot be relaxed to the weaker condition that it is a rational function. We also compute the Hilbert series of the algebra of invariants of the multiplicative group of the base field acting on the 2-generated relatively free algebra in the variety generated by the $2 \times 2$ matrix algebra.

Finally, we change our topic in Section 7, and consider group actions on PIalgebras (algebras satisfying a polynomial identity), that are not necessarily relatively free algebras. In fact a finitely generated associative $K$-algebra is a PI-algebra if and only if it is a homomorphic image of some $F_{n}(\Re)$ for some $n$ and a proper subvariety $\mathfrak{R}$ of the variety of associative algebras. Vonessen 31] proved that if $G$ is a linearly reductive group acting rationally on a finitely generated noetherian PI-algebra $R$, then the subalgebra $R^{G}$ is finitely generated and noetherian. We note here that the transfer principle (a well-known tool in commutative invariant theory, see [19]) can be applied in this noncommutative setting as well, and assert in Theorem 7.2 that under the conditions in the theorem of Vonessen, $R^{H}$ is finitely generated and noetherian for any subgroup $H$ of $G$ for which $K[G]^{H}$ is finitely generated, where the action of $H$ on the coordinate ring $K[G]$ of $G$ is induced by the right translation action of $H$ on $G$.

We close the introduction by mentioning that a more general setup for developing noncommutative invariant theory that also received attention is provided by Hopf algebra actions on noncommutative rings, see for example the survey [3], the papers [20], 15, and the references therein. A typical problem considered in these works is whether the existence of a polynomial identity for the algebra of invariants implies the existence of an identity for the whole algebra.

\section{A CHARACTERIZATION OF NICE RATIONAL SYMMETRIC FUNCTIONS}

Write $G L_{n}$ for the general linear group $G L_{n}(K)$ over $K$, viewed as an affine algebraic group. In this paper by a representation of $G L_{n}$ we shall always mean a rational representation, i.e., a $K$-vector space $Z$ together with a group homomorphism $\rho$ : $G L_{n} \rightarrow G L(Z)$ giving an action of $G L_{n}$ on $Z$ via linear transformations such that $Z$ is spanned by finite dimensional $G L_{n}$-invariant subspaces and for any finite dimensional $G L_{n}$-invariant subspace $W$ of $Z$, the map $G L_{n} \rightarrow G L(W),\left.g \mapsto \rho(g)\right|_{W}$ is a morphism of affine algebraic varieties. When $Z$ is finite dimensional, define the formal character of $Z$ as the element $\operatorname{ch}_{Z}$ in the Laurent polynomial ring $\mathbb{Z}\left[t_{1}^{ \pm 1}, \ldots, t_{n}^{ \pm 1}\right]$ satisfying that for any diagonal matrix $\operatorname{diag}\left(z_{1}, \ldots, z_{n}\right) \in G L_{n}$ with diagonal entries $z_{1}, \ldots, z_{n} \in K \backslash\{0\}$ we have the equality $\operatorname{Tr}\left(\rho\left(\operatorname{diag}\left(z_{1}, \ldots, z_{n}\right)\right)\right)=\operatorname{ch}_{V}\left(z_{1}, \ldots, z_{n}\right)$. In fact $\operatorname{ch}_{V} \in \mathbb{N}_{0}\left[t_{1}^{ \pm 1}, \ldots, t_{n}^{ \pm 1}\right]$ has non-negative coefficients and is symmetric in the variables $t_{1}, \ldots, t_{n}$. Hence it belongs to the subring $\mathbb{Z}\left[t_{1}^{ \pm 1}, \ldots, t_{n}^{ \pm 1}\right]^{S_{n}}$ of $S_{n^{-}}$ invariants in $\mathbb{Z}\left[t_{1}^{ \pm 1}, \ldots, t_{n}^{ \pm 1}\right]$, where the symmetric group $S_{n}$ acts by permuting the indeterminates. It is well known that for any sequence $\lambda \in \mathbb{Z}^{n}$ with $\lambda_{1} \geq \cdots \geq \lambda_{n}$ there is a finite dimensional representation $L_{\lambda}$ of $G L_{n}$ whose formal character is given by the equality

$$
\operatorname{ch}_{L_{\lambda}} \cdot \prod_{1 \leq i<j \leq n}\left(t_{i}-t_{j}\right)=\sum_{\sigma \in S_{n}} \operatorname{sign}(\sigma) \prod_{i=1}^{n} t_{\sigma(i)}^{\lambda_{i}+n-i} .
$$

The set of all $\mathrm{ch}_{L_{\lambda}}$ forms a basis of the free $\mathbb{Z}$-module $\mathbb{Z}\left[t_{1}^{ \pm 1}, \ldots, t_{n}^{ \pm 1}\right]^{S_{n}}$. Note that when $\lambda_{n} \geq 0$, the formal character $\operatorname{ch}_{L_{\lambda}}$ is the Schur function associated to the partition $\lambda$. Moreover, all representations of $G L_{n}$ are completely reducible, and 
$\left\{L_{\lambda} \mid \lambda \in \mathbb{Z}^{n}, \lambda_{1} \geq \cdots \geq \lambda_{n}\right\}$ is a complete irredundant list of representatives of the isomorphism classes of irreducible representations of $G L_{n}$ (see for example Section 9.8.1 in [29]). We shall deal with the situation when $Z=\bigoplus_{d=0}^{\infty} Z_{d}$ is graded, each homogeneous component $Z_{d}$ is finite dimensional and $G L_{n}$-invariant. In this case we say that $Z$ is a graded representation of $G L_{n}$ and call the formal character of the graded representation $Z$ the formal power series

$$
\operatorname{ch}_{Z}(q)=\sum_{d=0}^{\infty} \operatorname{ch}_{Z_{d}} \cdot q^{d} \in \mathbb{Z}\left[t_{1}^{ \pm 1}, \ldots, t_{n}^{ \pm 1}\right]^{S_{n}}[[q]] .
$$

Definition 2.1. Denote by $\mathcal{C}$ the class of graded representations $Z$ of $G L_{n}$ that have also the structure of a finitely generated graded module over the symmetric tensor algebra (polynomial algebra) $S(W)$ for some finite dimensional graded representation $W$ of $G L_{n}$ (the grading on $W$ induces a grading on $S(W)$ in the standard way), such that the action of $G L_{n}$ on $Z$ and $S(W)$ is compatible with the $S(W)$-module structure of $V$ (i.e., for $g \in G L_{n}, f \in S(W)$, and $m \in Z$, we have $g \cdot(f m)=(g \cdot f)(g \cdot m))$.

Proposition 2.2. An element $f \in \mathbb{Z}\left[t_{1}^{ \pm 1}, \ldots, t_{n}^{ \pm 1}\right]^{S_{n}}[[q]]$ belongs to the $\mathbb{Z}$-submodule of $\mathbb{Z}\left[t_{1}^{ \pm 1}, \ldots, t_{n}^{ \pm 1}\right]^{S_{n}}[[q]]$ generated by $\left\{\operatorname{ch}_{V}(q) \mid V \in \mathcal{C}\right\}$ if and only if $f$ is of the form

$$
f=\frac{P\left(t_{1}, \ldots, t_{n}, q\right)}{\prod_{(\alpha, k)}\left(1-t_{1}^{\alpha_{1}} \cdots t_{n}^{\alpha_{n}} q^{k}\right)}
$$

where $P \in \mathbb{Z}\left[t_{1}^{ \pm 1}, \ldots, t_{n}^{ \pm 1}\right][q]$ is a polynomial in $q$ and the product in the denominator ranges over a finite multiset of pairs $(\alpha, k)$ where $\alpha \in \mathbb{Z}^{n}, k \in \mathbb{N}_{0}$.

Proof. For $\lambda \in \mathbb{Z}^{n}$ with $\lambda_{1} \geq \cdots \geq \lambda_{n}$ and $k \in \mathbb{N}_{0}$ write

$$
e_{k}^{\lambda}=\prod\left(1-t_{1}^{\alpha_{1}} \cdots t_{n}^{\alpha_{n}} q^{k}\right)
$$

where a factor $\left(1-t_{1}^{\alpha_{1}} \cdots t_{n}^{\alpha_{n}} q^{k}\right)$ in the above product occurs exactly with multiplicity the coefficient of $t_{1}^{\alpha_{1}} \cdots t_{n}^{\alpha_{n}}$ in $\operatorname{ch}_{L_{\lambda}} \in \mathbb{N}_{0}\left[t_{1}^{ \pm 1}, \ldots, t_{n}^{ \pm 1}\right]$.

Suppose that $f$ is of the form (11). For any $\alpha \in \mathbb{Z}^{n}$ and $k \in \mathbb{N}_{0},\left(1-t_{1}^{\alpha_{1}} \cdots t_{n}^{\alpha_{n}} q^{k}\right)$ is a factor of $e_{k}^{\lambda}$, where $\lambda$ is obtained by rearranging the elements in the sequence $\left(\alpha_{1}, \ldots, \alpha_{n}\right)$ in the decreasing order. Therefore we may assume that the denominator of $f$ in (10) is $\prod_{(\lambda, k) \in A} e_{k}^{\lambda}$ for a finite multiset $A$ of pairs $(\lambda, k)$ where $k \in \mathbb{N}_{0}$, $\lambda \in \mathbb{Z}^{n}$ with $\lambda_{1} \geq \cdots \geq \lambda_{n}$. Then the denominator of $f$ is symmetric, hence the numerator $P$ of $f$ belongs to $\mathbb{Z}\left[t_{1}^{ \pm 1}, \ldots, t_{n}^{ \pm 1}\right]^{S_{n}}[q]$ and can be written as an integral linear combination

$$
P=\sum_{(\mu, r) \in B} m_{\mu, r} \operatorname{ch}_{L_{\mu}}\left(t_{1}, \ldots, t_{n}\right) q^{r}
$$

where $B$ is a finite multiset of pairs $(\mu, r), r \in \mathbb{N}_{0}, \mu \in \mathbb{Z}^{n}, \mu_{1} \geq \cdots \geq \mu_{n}$. Take the finite dimensional graded $G L_{n}$-representation

$$
W=\bigoplus W_{k}, \quad W_{k}=\bigoplus_{\{\lambda \mid(\lambda, k) \in A\}} L_{\lambda} .
$$


By construction of $W$ the graded $G L_{n}$-representation $S(W)$ has formal character

$$
\operatorname{ch}_{S(W)}(t)=\frac{1}{\prod_{(\lambda, k) \in A} e_{k}^{\lambda}} .
$$

For $(\mu, r) \in B$ denote by $L_{\mu}^{[r]}$ the finite dimensional graded $G L_{n}$-representation whose degree $r$ component is $L_{\mu}$ and all other homogeneous components are zero; the formal character of this graded representation is $q^{r} \mathrm{ch}_{L_{\mu}}\left(t_{1}, \ldots, t_{n}\right)$. Consider now the graded $G L_{n}$-representation $S(W) \otimes L_{\mu}^{[r]}$. It is naturally a graded $S(W)$ module, it is free and is generated by the finite dimensional subspace $1 \otimes L_{\mu}^{[r]}$. Moreover, the $G L_{n}$-action on $S(W)$ is compatible with the action on $S(W) \otimes L_{\mu}^{[r]}$. Thus $S(W) \otimes L_{\mu}^{[r]}$ belongs to $\mathcal{C}$, and

$$
\operatorname{ch}_{S(W) \otimes L_{\mu}^{[r]}}(q)=\operatorname{ch}_{S(W)}(q) \cdot \operatorname{ch}_{L_{\mu}^{[r]}}(q)=\frac{\operatorname{ch}_{L_{\mu}}\left(t_{1}, \ldots, t_{n}\right) q^{r}}{\prod_{(\lambda, k) \in A} e_{k}^{\lambda}} .
$$

Summarizing, we obtained

$$
f=\frac{\sum_{(\mu, r) \in B} m_{\mu, r} \operatorname{ch}_{L_{\mu}}\left(t_{1}, \ldots, t_{n}\right) q^{r}}{\prod_{(\lambda, k) \in A} e_{k}^{\lambda}}=\sum_{(\mu, r) \in B} m_{\mu, r} \operatorname{ch}_{S(W) \otimes L_{\mu}^{[r]}}
$$

showing that $f$ belongs to $\sum_{Z \in \mathcal{C}} \mathbb{Z} \operatorname{ch}_{Z}$.

To see the converse, take $Z \in \mathcal{C}$. Then $Z$ is a finitely generated graded module over the commutative polynomial algebra $S(W)$, where $W=\bigoplus_{k=0}^{\infty} W_{k}$ is a finite dimensional graded representation of $G L_{n}$. By the Hilbert Syzygy Theorem, there exists a $d \leq \operatorname{dim}(W)$ and an exact sequence

$$
0 \rightarrow F_{d} \rightarrow F_{d-1} \rightarrow \cdots \rightarrow F_{1} \rightarrow F_{0} \rightarrow Z \rightarrow 0
$$

of graded $S(W)$-module homomorphisms, where the $F_{i}$ are of the form $S(W) \otimes U_{i}$ for some finite dimensional graded representations $U_{i}$ of $G L_{n}$ (so they are free $S(W)$ modules belonging to $\mathcal{C}$ ), and the maps are $G L_{n}$-equivariant. Indeed, take for $U_{0}$ a $G L_{n}$-stable direct complement of $S(W)_{+} Z$, where $S(W)_{+}$stands for the sum of the positive degree homogeneous components of $S(W)$. Since $S(W)_{+} Z$ is a graded subspace of $Z$, we may also assume that $U_{0}$ is spanned by homogeneous elements. Then $U_{0}$ minimally generates $Z$ as an $S(W)$-module and is finite dimensional by the graded Nakayama Lemma (see for example Lemma 3.5.1 in [7]). So we get an $S(W)$-module surjection $\varphi_{0}: S(W) \otimes U_{0} \rightarrow Z$, which is $G L_{n}$-equivariant by construction. Moreover, identifying the subspace $1 \otimes U_{0}$ of $S(W) \otimes U_{0}$ with $U_{0} \subset Z$, the free module $S(W) \otimes U_{0}$ becomes graded, and the surjection $\varphi_{0}$ is a homomorphism of graded modules. Next repeat the same construction for the kernel of the homomorphism $\varphi_{0}$ instead of $Z$, to come up with $U_{1}$ and $\varphi_{1}: S(W) \otimes U_{1} \rightarrow \operatorname{ker}\left(\varphi_{0}\right)$. Continue in the same way. This process stops in at most $\operatorname{dim}(W)$ steps by the Hilbert Syzygy Theorem (see for example Section 1.3.2 in [7]), and we obtain the desired exact sequence (2). It follows that $\operatorname{ch}_{M}(q)=\sum_{j=0}^{d}(-1)^{j} \operatorname{ch}_{F_{i}}(q)$. Note that 
$\operatorname{ch}_{F_{i}}=\operatorname{ch}_{S(W)}(q) \cdot \operatorname{ch}_{U_{i}}(q) \cdot \operatorname{Here}_{U_{i}}(q) \in \mathbb{Z}\left[t_{1}^{ \pm 1}, \ldots, t_{n}^{ \pm 1}\right]^{S_{n}}[q]$ is a polynomial in $q$, whereas $\operatorname{ch}_{S(W)}(q)=\prod_{(\lambda, k)}\left(e_{k}^{\lambda}\right)^{-m_{\lambda, k}}$, where $m_{\lambda, k}$ stands for the multiplicity of $L_{\lambda}$ as a summand in $W_{k}$. It follows that

$$
\operatorname{ch}_{Z}(q)=\frac{\sum_{i=0}^{d}(-1)^{i} \operatorname{ch}_{U_{i}}(q)}{\prod_{(\lambda, k)}\left(e_{k}^{\lambda}\right)^{m_{\lambda, k}}}
$$

is indeed of the form (11).

Definition 2.3. Following Berele [5] (but changing a bit the setup considered by him) we call an element of $\mathbb{Z}\left[t_{1}^{ \pm 1}, \ldots, t_{n}^{ \pm 1}\right][[q]]$ a nice rational function if it is of the form (1).

Remark 2.4. (i) With this terminology Proposition 2.2 asserts that the abelian group of symmetric nice rational functions is generated by $\left\{\operatorname{ch}_{V}(q) \mid V \in \mathcal{C}\right\}$.

(ii) Nice rational functions not depending on the indeterminates $t_{1}, \ldots, t_{n}$ are rational functions in $\mathbb{Z}[[q]]$ with denominators which are products of binomials $1-q^{k}$. Recall that the Hilbert-Serre Theorem asserts that the Hilbert series of a finitely generated graded module over a finitely generated commutative graded $K$-algebra $R$ with $R_{0}=K$ is a nice rational function in $\mathbb{Z}[[q]]$.

(iii) If $f\left(t_{1}, \ldots, t_{n}\right)$ is a nice rational function in the sense of [5], then making the substitution $t_{i} \mapsto t_{i} q$ we get that $f\left(t_{1} q, \ldots, t_{n} q\right)$ is a nice rational function in the sense of our Defininition 2.3 .

\section{HILbERT SERIES OF FIXED POINT SUBSPACES}

Fix now a subgroup $G$ of $G L_{n}$. We write $Z^{G}$ for the subspace of $G$-fixed points in a representation $Z$ of $G L_{n}$. Define

$$
D: \mathbb{Z}\left[t_{1}^{ \pm 1}, \ldots, t_{n}^{ \pm 1}\right]^{S_{n}} \rightarrow \mathbb{Z}
$$

as the abelian group homomorphism with $D\left(\operatorname{ch}_{L_{\lambda}}\right)=\operatorname{dim}\left(L_{\lambda}^{G}\right)$. This definition makes sense, since the $\operatorname{ch}_{L_{\lambda}}$ constitute a free $\mathbb{Z}$-module basis in $\mathbb{Z}\left[t_{1}^{ \pm 1}, \ldots, t_{n}^{ \pm 1}\right]^{S_{n}}$. Furthermore, we keep the notation $D$ for the abelian group homomorphism

$$
D: \mathbb{Z}\left[t_{1}^{ \pm 1}, \ldots, t_{n}^{ \pm 1}\right]^{S_{n}}[[q]] \rightarrow \mathbb{Z}[[q]] \text { with } D\left(\sum_{d=0}^{\infty} c_{d} q^{d}\right)=\sum_{d=0}^{\infty} D\left(c_{d}\right) q^{d}
$$

Since every representation of $G L_{n}$ is completely reducible, for any finite dimensional $G L_{n}$-representation $W$ we have $D\left(\operatorname{ch}_{W}\right)=\operatorname{dim} W^{G}$, and consequently for a graded representation $Z=\bigoplus_{d=0}^{\infty} Z_{d}$ of $G L_{n}$ we have that

$$
D\left(\operatorname{ch}_{Z}(q)\right)=\sum_{d=0}^{\infty} \operatorname{dim}\left(Z_{d}^{G}\right) q^{d}=H\left(Z^{G}, q\right)
$$

is the Hilbert series of the graded vector space $Z^{G}$.

We need the following technical lemma (we provide a proof, since though the arguments are well known, we did not find a reference where this is stated exactly in the form as below):

Lemma 3.1. The following conditions are equivalent for a subgroup $G$ of $G L_{n}$ : 
(i) For any $Z \in \mathcal{C}$, the subspace $Z^{G}$ is finitely generated as an $S(W)^{G}$-module (where $W$ is as in Definition 2.1 of the class $\mathcal{C}$ ).

(ii) For any finitely generated graded commutative $K$-algebra $R$ on which $G L_{n}$ acts rationally via graded $K$-algebra automorphisms, the subalgebra $R^{G}$ of $G$-invariants is a finitely generated $K$-algebra.

Proof. Suppose (i) holds, and take a $K$-algebra $R$ as in (ii). Denote by $R_{+}$the sum of the positive degree homogeneous components in $R$. Then $R_{+}$contains a finite dimensional $G L_{n}$-submodule $W$ such that $W$ is spanned by homogeneous elements, and $W$ generates $R$ as a $K$-algebra. The identity map $W \rightarrow W$ extends to a surjection $S(W) \rightarrow R$ of graded $K$-algebras, and this surjection is $G$-equivariant. So in this way $R_{+}$becomes a graded $S(W)$-module in the class $\mathcal{C}$. Take a finite $S(W)^{G}$-module generating system of $R_{+}^{G}$ (it exists by assumption). This is easily seen to be a finite $K$-algebra generating system of $R^{G}$ by the graded Nakayama Lemma (see for example Lemma 3.5.1 in [7]).

Assume next that (ii) holds, and let $Z \in \mathcal{C}$. We repeat the proof of Theorem 16.9 in [19]: make the direct sum $R=S(W) \oplus Z$ a graded $K$-algebra by imposing the multiplication $(r, v) \cdot(s, w)=(r s, r w+s v)$ for $r, s \in S(W)$ and $v, w \in Z$. By assumption the algebra $R^{G}=S(W)^{G} \oplus Z^{G}$ is finitely generated, implying in turn that the $S(W)^{G}$-module $Z^{G}$ is finitely generated.

Remark 3.2. The equivalent conditions (i) and (ii) of Lemma 3.1 hold for a subgroup $G$ of $G L_{n}$ if and only if they hold for its Zariski closure $\bar{G}$ in $G L_{n}$. Indeed, for a given element $f$ of an algebra $R$ as in (ii), the stabilizer of $f$ in $G L_{n}$ is Zariski closed, since $G L_{n}$ acts rationally on $R$. Therefore we have $R^{G}=R^{\bar{G}}$.

We mention two important and well-known sufficient conditions that imply condition (ii) (and hence condition (i)) of Lemma 3.1

Proposition 3.3. The conditions (i) and (ii) of Lemma 3.1 hold for G in each of the following two cases:

(1) $G$ is a Zariski closed reductive subgroup of $G L_{n}$.

(2) $G$ is the unipotent radical of a Borel subgroup of a Zariski closed reductive subgroup of $G L_{n}$.

Proof. (1) is classical, see for example Theorem A (a) on page 3 of [19] and the reference therein. (2) is due to Hadžiev [21] and Grosshans (in arbitrary characteristic, see Theorem 9.4 in [19]).

Theorem 3.4. Suppose that the subgroup $G$ of $G L_{n}$ satisfies condition (i) (and hence (ii)) of Lemma 3.1, and let $Z$ be a graded representation of $G L_{n}$ for which $\operatorname{ch}_{Z}(q)$ is a nice rational function in the sense of Definition 2.3. Then the Hilbert series $H\left(Z^{G}, q\right) \in \mathbb{Z}[[q]]$ of the subspace of $G$-invariants in $Z$ is a rational function of the form

$$
H\left(Z^{G}, q\right)=\frac{P(q)}{\prod_{j=1}^{m}\left(1-q^{d_{j}}\right)}
$$

for some positive integers $m, d_{1}, \ldots, d_{m}$ and a polynomial $P \in \mathbb{Z}[q]$.

Proof. Since $\operatorname{ch}_{Z}(q)$ is a nice rational function and it is also symmetric in $t_{1}, \ldots, t_{n}$, by Proposition 2.2 there exists a positive integer $r, Z_{1}, \ldots, Z_{r} \in \mathcal{C}$ and integers 
$a_{1}, \ldots, a_{r}$ such that $\operatorname{ch}_{Z}(q)=\sum_{i=1}^{r} a_{i} \operatorname{ch}_{Z_{i}}$. By (3) we have

$$
H\left(Z^{G}, q\right)=D\left(\operatorname{ch}_{Z}(q)\right)=\sum_{i=1}^{r} a_{i} D\left(\operatorname{ch}_{Z_{i}}\right)=\sum_{i=1}^{r} a_{i} H\left(Z_{i}^{G}, q\right) .
$$

Here $Z_{i}$ is a finitely generated graded $S\left(W_{i}\right)$-module (with compatible $G L_{n}$-action) for some finite dimensional graded representation $W_{i}$ of $G L_{n}, i=1, \ldots, r$. Since conditions (i) and (ii) of Lemma 3.1 hold for $G$, we have that the algebra $S\left(W_{i}\right)^{G}$ is finitely generated, and $Z_{i}^{G}$ is a finitely generated graded $S\left(W_{i}\right)^{G}$-module for $i=1, \ldots, r$. By the Hilbert-Serre Theorem (see for example Theorem 11.1 in [2]) the finitely generated $S\left(W_{i}\right)^{G}$-module $Z_{i}^{G}$ has a rational Hilbert series $H\left(Z_{i}^{G}, q\right)$ of the stated form, implying in turn by (4) that $H\left(Z^{G}, q\right)$ is a rational function of the stated form (i.e. a nice rational function in $\mathbb{Z}[[q]]$ ).

\section{Examples in COMmutative AlgEBRA}

We do not know any example of an algebra of commutative polynomial invariants with Hilbert series which is not a rational function. In this section we compute the Hilbert series of a famous algebra of invariants which is not finitely generated but as it turns out, still has a nice rational Hilbert series. The first example of a finite dimensional $G$-module $V$ such that the corresponding algebra $K[V]^{G}=$ $K\left[x_{1}, \ldots, x_{n}\right]^{G}$ of invariants is not finitely generated was found by Nagata (see [27]). A simplification of Nagata's argument (resulting in an example with a group of smaller dimension) was given by Steinberg [30. Here we shall compute the Hilbert series of this latter algebra of invariants. We refer to the books [8], 26] for facts about this algebra of invariants.

Let $C$ be an irreducible cubic algebraic curve in the complex projective plane $\mathbb{P}^{2}$. The set of non-singular points in $C$ has a well-known abelian group structure. Choose 9 distinct points $\left(a_{i 1}: a_{i 2}: a_{i 3}\right)$ in this abelian group such that their sum is not a torsion element and the matrix $\left(a_{i j}\right)_{i=1, \ldots, 9}^{j=1,2,3}$ has full rank 3 . Let $G_{1}$ be the subgroup of $\mathbb{C}^{9}$ (the direct sum of 9 copies of the additive group of $\mathbb{C}$ ) given by

$$
G_{1}=\left\{s=\left(s_{1}, \ldots, s_{9}\right) \mid \sum_{i=1}^{9} s_{i} a_{i j}=0, \quad j=1,2,3\right\} \subset \mathbb{C}^{9}
$$

so $G \cong \mathbb{C}^{6}$. Consider also the torus

$$
T=\left\{t=\left(t_{1}, \ldots, t_{9}\right) \mid \prod_{i=1}^{9} t_{i}=1\right\} \subset\left(\mathbb{C}^{\times}\right)^{9}
$$

so $T \cong\left(\mathbb{C}^{\times}\right)^{8}$. The group $G=G_{1} \times T \subset \mathbb{C}^{9} \times\left(\mathbb{C}^{\times}\right)^{9}$ acts on the 18 -variable polynomial algebra $S=\mathbb{C}\left[x_{1}, \ldots, x_{9}, y_{1}, \ldots, y_{9}\right]$ via

$$
(s, t) \cdot x_{i}=t_{i} x_{i}, \quad(s, t) \cdot y_{i}=t_{i}\left(s_{i} x_{i}+y_{i}\right), \quad i=1, \ldots, 9 .
$$

Set

$$
A_{j}=\sum_{i=1}^{9} a_{i j} \frac{y_{i}}{x_{i}}, \quad j=1,2,3, \quad D=x_{1} \cdots x_{9} .
$$

Then $A_{1}, A_{2}, A_{3}, D$ generate the subfield of $G$-invariants in the field of fractions of $S$, and in fact

$$
R=S^{G}=S \cap \mathbb{C}\left[A_{1}, A_{2}, A_{3}, D, D^{-1}\right]
$$


Thus $R$ has a bigrading $R=\bigoplus_{d \in \mathbb{N}_{0}, m \in \mathbb{Z}} R_{(d, m)}$, where

$$
R_{(d, m)}=S \cap\left\{D^{d-m} f\left(A_{1}, A_{2}, A_{3}\right) \mid f \in \mathbb{C}[x, y, z] \text { is homogeneous of degree } d\right\} .
$$

Theorem 4.1. (i) (Proposition 2.49 in [26] or page 60 in [8]) The $\mathbb{C}$-algebra $R=S^{G}$ is not finitely generated.

(ii) (Lemma 2.50 in [26] and Lemma 4.5 in [8]) For $d \in \mathbb{N}_{0}$ and $m \in \mathbb{Z}$ we have

$$
\operatorname{dim} R_{(d, m)}= \begin{cases}\left(\begin{array}{c}
d+2 \\
2 \\
d+2 \\
2
\end{array}\right)-9\left(\begin{array}{c}
m+1 \\
2
\end{array}\right), & \text { if } \left.\quad \begin{array}{l} 
\\
2
\end{array}\right)=3 m>0 ; \\
0, & \text { if } \quad 3 m>d .\end{cases}
$$

Observe that $R_{(d, m)}$ is contained in the homogeneous component of degree $9(d-$ $m$ ) of the polynomial algebra of $S$, hence we have

$$
H(S, q)=\sum_{n=0}^{\infty} c_{n} q^{9 n} \text { where } c_{n}=\sum_{m \geq-n} \operatorname{dim} R_{(m+n, m)} .
$$

We infer from Theorem 4.1 (ii) that

$$
c_{n}=\sum_{m=1}^{\left\lfloor\frac{n}{2}\right\rfloor}\left(\left(\begin{array}{c}
n+m+2 \\
2
\end{array}\right)-9\left(\begin{array}{c}
m+1 \\
2
\end{array}\right)\right)+\sum_{m=-n}^{0}\left(\begin{array}{c}
n+m+2 \\
2
\end{array}\right)
$$

where $\left\lfloor\frac{n}{2}\right\rfloor$ stands for the lower integer part of $\frac{n}{2}$. Now we have

$$
\sum_{m=-n}^{0}\left(\begin{array}{c}
n+m+2 \\
2
\end{array}\right)=\left(\begin{array}{c}
n+3 \\
3
\end{array}\right)
$$

for $n=2 s>0$ even we have

$$
\sum_{m=1}^{s} \operatorname{dim} R_{(2 s+m, m)}=\frac{1}{6}\left(10 s^{3}+3 s^{2}-7 s\right)=10\left(\begin{array}{l}
s \\
3
\end{array}\right)+11\left(\begin{array}{l}
s \\
2
\end{array}\right)+s,
$$

and for $n=2 s+1$ odd we have

$$
\sum_{m=1}^{s} \operatorname{dim} R_{(2 s+1+m, m)}=\frac{1}{6}\left(10 s^{3}+18 s^{2}+8 s\right)=10\left(\begin{array}{l}
s \\
3
\end{array}\right)+16\left(\begin{array}{l}
s \\
2
\end{array}\right)+6 s .
$$

We obtain

$$
\begin{aligned}
\sum_{n=0}^{\infty} c_{n} q^{n} & =\sum_{n=0}^{\infty}\left(\begin{array}{c}
n+3 \\
3
\end{array}\right) q^{n} \\
& +\sum_{s=1}^{\infty}\left(10\left(\begin{array}{l}
s \\
3
\end{array}\right)+11\left(\begin{array}{l}
s \\
2
\end{array}\right)+s\right) q^{2 s} \\
& +\sum_{s=0}^{\infty}\left(10\left(\begin{array}{l}
s \\
3
\end{array}\right)+16\left(\begin{array}{l}
s \\
2
\end{array}\right)+6 s\right) q^{2 s+1} \\
& =\frac{1}{(1-q)^{4}}+\frac{10 q^{6}+10 q^{7}}{\left(1-q^{2}\right)^{4}}+\frac{11 q^{4}+16 q^{5}}{\left(1-q^{2}\right)^{3}}+\frac{q^{2}+6 q^{3}}{\left(1-q^{2}\right)^{2}} \\
& =\frac{1+4 q+7 q^{2}+10 q^{3}+10 q^{4}+4 q^{5}}{\left(1-q^{2}\right)^{4}} .
\end{aligned}
$$


Summarizing, we found the following:

Proposition 4.2. We have the equality

$$
H\left(S^{G}, q\right)=\frac{1+4 q^{9}+7 q^{18}+10 q^{27}+10 q^{36}+4 q^{45}}{\left(1-q^{18}\right)^{4}} .
$$

In particular, $H\left(S^{G}, q\right)$ is a nice rational function.

Problem 4.3. Does there exist a $G$-module $V$ such that $K[V]^{G}=K\left[x_{1}, \ldots, x_{n}\right]^{G}$ has a non-rational Hilbert series?

There are many examples of subalgebras of the polynomial algebra in several variables which are not finitely generated. See e.g., 28] for a family of subalgebras between $K\left[x_{1}\right]$ and $K\left[x_{1}, x_{2}\right]$ which are not finitely generated as $K\left[x_{1}\right]$-algebras. It is also easy to construct a graded subalgebra of $K\left[x_{1}, x_{2}\right]$ with transcendental Hilbert series. One of the few general abstract properties that all subalgebras of invariants in the polynomial algebra do have is that they are normal (integrally closed in their field of fractions). Below we give an example showing that normality does not imply rationality of the Hilbert series.

Example 4.4. Fix a positive irrational real number $\alpha$ and consider the subalgebra $A=\operatorname{Span}_{K}\left\{x_{1}^{i} x_{2}^{j} \mid j<\alpha i\right\}$ of $K\left[x_{1}, x_{2}\right]$. The algebra $A$ is not finitely generated (see for example [28]). The number of degree $d \geq 1$ monomials in $A$ is $\mid\{i \mid i \leq$ $d, \quad d-i<\alpha i\} \mid$, which is the number of integers in the interval $\left(\frac{d}{1+\alpha}, d\right]$. Set $\beta=1-\frac{1}{1+\alpha}=\frac{\alpha}{\alpha+1}$. The Hilbert series of $A$ is

$$
H(A, q)=1+\sum_{d=1}^{\infty}(1+\lfloor d \beta\rfloor) q^{d}=\frac{1}{1-q}+\sum_{d=1}^{\infty}\lfloor d \beta\rfloor q^{d},
$$

where $\lfloor d \beta\rfloor$ stands for the lower integer part of $d \beta$. Suppose that this power series is algebraic over $\mathbb{Z}(q)$. Then $H_{1}(q)=\sum_{d=1}^{\infty}\lfloor d \beta\rfloor q^{d}$ is algebraic as well, implying in turn that $H_{2}(q)=(1-q) H_{1}(q)=\sum_{d=1}^{\infty}(\lfloor d \beta\rfloor-\lfloor(d-1) \beta\rfloor) q^{d}$ is algebraic. Since $0<\beta<1$, we have $c_{d}=\lfloor d \beta\rfloor-\lfloor(d-1) \beta\rfloor \in\{0,1\}$, so the coefficients of $H_{2}(q)$ take only two integer values. By the classical theorem of Fatou [16], see Borwein and Coons [6] for an easy proof, $H_{2}(q)$ must be a rational function. As in the proof of Theorem 2 in [6], this implies that the sequence $\left(c_{d} \mid d=1,2, \ldots\right)$ is eventually periodic. This contradicts the assumption that $\beta$ is irrational. Indeed, suppose that there exist positive integers $N, p$ such that $c_{d+p}=c_{d}$ for all $d \geq N$. Set $s=\left|\left\{1 \leq i \leq p \mid c_{N+i}=1\right\}\right|$. Then for any positive integer $k$ we have

$$
\lfloor(N+k p) \beta\rfloor-\lfloor N \beta\rfloor=\sum_{i=N+1}^{N+k p} c_{i}=k s .
$$

We conclude that $|k p \beta-k s| \leq 1$, and hence $\left|\beta-\frac{s}{p}\right| \leq \frac{1}{k p}$ holds for all $k$. This leads to the contradiction $\beta=\frac{s}{p}$. 
We claim that $A$ is normal. Indeed, note that $A$ is the semigroup algebra of the subsemigroup $S=\{(i, j) \mid j \leq \alpha i\}$ of the additive semigroup $\mathbb{N}_{0}^{2}$. The semigroup $S$ is saturated in $\mathbb{N}_{0}^{2}$, i.e., if $m s \in S$ for some $s \in \mathbb{N}_{0}^{2}$ and $m \in \mathbb{N}$ then $s \in S$. Suppose that some element $f$ from the field of fractions of $A$ is integral over $A$. Then there exists a finitely generated subsemigroup $S^{\prime} \subset S$ such that $f$ is integral over the subalgebra $K\left[S^{\prime}\right]=\operatorname{Span}_{K}\left\{x_{1}^{i} x_{2}^{j} \mid(i, j) \in S^{\prime}\right\}$ of $A$ (take all monomials that occur in the coefficients of the monic polynomial in $A[x]$ satisfied by $f$ ). So $f$ belongs to the normalization of $K\left[S^{\prime}\right]$, which is $K\left[S_{\text {sat }}^{\prime}\right]$, where $S_{\text {sat }}^{\prime}=\mathbb{Q} \geq 0 S^{\prime} \cap \mathbb{N}_{0}^{2}$ is the saturation of $S^{\prime}$ (see for example Proposition 7.25 in [25]). Now since $S$ is saturated, we have $S_{\text {sat }}^{\prime} \subset S$, so $f \in K[S]=A$, showing that $A$ is integrally closed in its field of fractions.

\section{Applications in noncommutative invariant theory}

Let $\mathfrak{R}$ be a proper subvariety of the variety of all associative $K$-algebras. Denote by $F_{n}(\mathfrak{R})$ the relatively free algebra of rank $n$ in the variety $\mathfrak{R}$. Then $F_{n}(\mathfrak{R})=$ $K\langle V\rangle / I$ is the factor algebra of the tensor algebra $K\langle V\rangle$ of an $n$-dimensional vector space $V$ modulo a non-zero T-ideal $I$. Choosing a basis $\left\{x_{1}, \ldots, x_{n}\right\}$ in $V$ we identify $G L_{n}$ with $G L(V)$ and the tensor algebra $K\langle V\rangle$ with the free associative algebra $K\left\langle x_{1}, \ldots, x_{n}\right\rangle$. This is graded as usual, so the generators $x_{i}$ have degree 1. Since $I$ is necessarily homogeneous, there is an induced grading on $F_{n}(\Re)$, and $F_{n}(\Re)=\bigoplus_{d=0}^{\infty} F_{n}(\Re)_{d}$ is a graded representation of $G L_{n}$ in the sense of Section 2 For any subgroup $G \leq G L_{n}$, the subalgebra $F_{n}(\mathfrak{R})^{G}=\bigoplus_{d=0}^{\infty} F_{n}(\mathfrak{R})_{d}^{G}$ is spanned by homogeneous elements, so it is also graded. Its Hilbert series is defined as

$$
H\left(F_{n}(\Re)^{G}, q\right)=\sum_{d=0}^{\infty} \operatorname{dim}\left(F_{n}(\Re)_{d}^{G}\right) q^{d} \in \mathbb{Z}[[q]] .
$$

Theorem 5.1. Suppose that condition (i) (and hence (ii)) of Lemma 3.1 holds for the subgroup $G$ of $G L_{n}$. Then the Hilbert series $H\left(F_{n}(\Re)^{G}, q\right)$ of the subalgebra of $G$-invariants in $F_{n}(\Re)$ is rational of the form

$$
H\left(F_{n}(\Re)^{G}, q\right)=\frac{P(q)}{\prod_{j=1}^{m}\left(1-q^{d_{j}}\right)}
$$

for some positive integers $m, d_{1}, \ldots, d_{m}$ and a polynomial $P \in \mathbb{Z}[q]$.

Proof. To simplify notation set $R=F_{n}(\mathfrak{R})$. An $\mathbb{N}_{0}^{n}$-grading on the free associative algebra $K\left\langle x_{1}, \ldots, x_{n}\right\rangle$ is defined by setting the multidegree of $x_{i}$ the $i$ th standard basis vector $(0, \ldots, 0,1,0, \ldots, 0)$ in $\mathbb{Z}^{n}$. This induces a multigrading $R=\bigoplus_{\alpha \in \mathbb{N}_{0}^{n}} R_{\alpha}$, and the multivariate Hilbert series of $R$ is

$$
H\left(R, t_{1}, \ldots, t_{n}\right)=\sum_{\alpha \in \mathbb{N}_{0}^{n}} \operatorname{dim}\left(R_{\alpha}\right) t_{1}^{\alpha_{1}} \cdots t_{n}^{\alpha_{n}} .
$$

Keep the notation $x_{i}$ for the image of $x_{i}$ under the natural surjection $K\left\langle x_{1}, \ldots, x_{n}\right\rangle \rightarrow$ $R$. Then there is a subset $S \subset\{1, \ldots, n\}^{d}$ such that the monomials $\left\{x_{i_{1}} \cdots x_{i_{d}}\right.$ | 
$\left.\left(i_{1}, \ldots, i_{d}\right) \in S\right\}$ constitute a basis of $R_{\alpha}$. Note that this basis of $R_{\alpha}$ consists of joint eigenvectors of the subgroup of diagonal matrices in $G L_{n}$, since we have

$$
\operatorname{diag}\left(z_{1}, \ldots, z_{n}\right) \cdot x_{i}=z_{i} x_{i}
$$

and consequently

$$
\operatorname{diag}\left(z_{1}, \ldots, z_{n}\right) \cdot x_{i_{1}} \cdots x_{i_{d}}=z_{1}^{\alpha_{1}} \cdots z_{n}^{\alpha_{n}} x_{i_{1}} \cdots x_{i_{d}} .
$$

For a fixed $d \in \mathbb{N}_{0}$ the degree $d$ homogeneous component $R_{d}$ of $R$ is $G L_{n}$-stable, and by the above considerations the sum of the terms of $H\left(R, t_{1}, \ldots, t_{n}\right)$ of total degree $d$ is the formal character $\operatorname{ch}_{R_{d}}$ of $R_{d}$. It follows that substituting $t_{i} \mapsto t_{i} q$ in the multivariate Hilbert series of $R$ we obtain

$$
H\left(R, q t_{1}, \ldots, q t_{n}\right)=\operatorname{ch}_{R}(q),
$$

the formal character of the graded $G L_{n}$-representation $R$. Belov 4 proved that the univariate Hilbert series $H(R, t)$ of the graded algebra $R$ is rational. Berele strengthened this statement in Theorem 1 of $[5$, by showing that the multigraded Hilbert series $H\left(R, t_{1}, \ldots, t_{n}\right)$ of $R$ is nice (in the sense of [5]), which implies that $H\left(R, q t_{1}, \ldots, q t_{n}\right)$ is a nice rational function in the sense of Definition 2.3. Summarizing, the formal character $\operatorname{ch}_{R}(q)$ is a nice rational function in the sense of Definition 2.3, thus the statement of our theorem now follows from Theorem 3.4.

Remark 5.2. In contrast with commutative invariant theory, the algebra $F_{n}(\mathfrak{R})^{G}$ is typically not finitely generated, even if condition (i) and (ii) of Lemma 3.1 hold for $G$. For example, by the proof of Theorem 3.1 in [11, if $\mathfrak{R}$ is not a subvariety of a variety of Lie nilpotent algebras with a given index of Lie nilpotency, then for any $n \geq 2$ there is a Zariski closed subgroup $G$ of $G L_{n}$ isomorphic to the multiplicative group of $K$ such that $F_{n}(\Re)^{G}$ is not finitely generated. Therefore Theorem 5.1yields interesting examples of naturally occurring graded algebras, that are not finitely generated, but have rational Hilbert series like finitely generated commutative graded algebras.

\section{EXAMPLES}

Example 6.1. We provide two examples showing that in Theorem 3.4 the condition that $\operatorname{ch}_{Z}(q)$ is a nice rational function can not be relaxed to the weaker condition that $\operatorname{ch}_{Z}(q)$ is a rational function.

(i) Let $V$ be 2-dimensional and $G=S L(V)$, the special linear subgroup of $G L(V)$. In this case $G$ is a Zariski closed reductive subgroup of $G L(V)$. The tensor algebra $K\langle V\rangle$ has rational Hilbert series

$$
H\left(K\langle V\rangle, t_{1}, t_{2}\right)=\frac{1}{1-t_{1}-t_{2}},
$$

whereas by Example 5.10 in [1] we have that the subalgebra of $S L(V)$-invariants has non-rational Hilbert series

$$
H\left(K\langle V\rangle^{S L(V)}, q\right)=\frac{1}{2 q^{2}}\left(1-\sqrt{1-4 q^{2}}\right)=\sum_{n=0}^{\infty} \frac{1}{n+1}\left(\begin{array}{c}
2 n \\
n
\end{array}\right) q^{n} .
$$

Further examples of subalgebras of $S L_{2}$-invariants in free algebras $K\left\langle x_{1}, \ldots, x_{n}\right\rangle$ having non-rational Hilbert series can be found in [1].

(ii) Again let $V=\operatorname{Span}_{K}\{x, y\}$ be 2-dimensional, and take

$$
G=\{x \mapsto x, y \mapsto c x+y \mid c \in K\} \subset G L(V) .
$$


In this case $G$ is the unipotent radical of a Borel subgroup of $G L(V)$, and in fact $G$ is isomorphic to the additive group of $K$. By Proposition 5.3 in [14] we have that

$$
\begin{gathered}
H\left((K\langle V\rangle)^{G}, q\right)=\sum_{p=0}^{\infty}\left(\left(\begin{array}{c}
2 p \\
p
\end{array}\right) q^{2 p}+\left(\begin{array}{c}
2 p+1 \\
p
\end{array}\right) q^{2 p+1}\right) \\
=\frac{1-\sqrt{1-4 q^{2}}}{2 q^{2}} \cdot \frac{1}{1-\frac{1-\sqrt{1-4 q^{2}}}{2 q}} .
\end{gathered}
$$

In particular, $H\left(K\langle V\rangle^{G}, q\right)$ is not rational.

Example 6.2. Here we compute the Hilbert series of a particular algebra of invariants in a relatively free algebra along the lines of the proof of Theorem 5.1 Let $V=\operatorname{Span}_{K}\{x, y\}$ be 2-dimensional, let $I$ be the T-ideal in $K\langle V\rangle$ of 2-variable identities of the algebra $K^{2 \times 2}$ of $2 \times 2$ matrices over $K$, and $G=K^{\times}$, the multiplicative group of the base field acting on $V$ via $z \cdot(a x+b y)=z a x+z^{-1} b y\left(z \in K^{\times}\right.$, $a, b \in K)$. The Hilbert series of $R=K\langle V\rangle / I$ was computed in [18]:

$$
H\left(R, t_{1}, t_{2}\right)=\frac{1}{\left(1-t_{1}\right)\left(1-t_{2}\right)}+\frac{t_{1} t_{2}}{\left(1-t_{1}\right)^{2}\left(1-t_{2}\right)^{2}\left(1-t_{1} t_{2}\right)} .
$$

Taking into account that $\operatorname{ch}_{R}(q)=H\left(R, t_{1} q, t_{2} q\right)$ we deduce from (5) the equality

$$
\operatorname{ch}_{R}(q)=\operatorname{ch}_{Z}(q)
$$

where $Z$ is the polynomial $G L(V)$-module

$$
Z=S(V) \oplus\left(\bigwedge^{2}(V) \otimes S(V \oplus V) \otimes S\left(\bigwedge^{2}(V)\right)\right)
$$

Here $\bigwedge^{2}(V)$ is the exterior tensor square of $V$, whereas $S(V)$ and $S(V \oplus V)$ are the symmetric tensor algebras of $V$ and $V \oplus V$. The grading is given by the decomposition into isotypic components with respect to the action of the subgroup of scalar transformations in $G L(V)$. Note that $G$ acts trivially on $\bigwedge^{2}(V)$, hence

$$
Z^{G}=S(V)^{G} \oplus\left(\bigwedge^{2}(V) \otimes S(V \oplus V)^{G} \otimes S\left(\bigwedge^{2}(V)\right)\right) .
$$

Clearly, $S(V)^{G}=K[x y]$ is a polynomial algebra generated by a degree 2 element, and $S(V \oplus V)^{G}=K\left[x_{1}, y_{1}, x_{2}, y_{2}\right]^{G}=K\left[x_{1} y_{1}, x_{1} y_{2}, x_{2} y_{1}, x_{2} y_{2}\right]$. It is well known that the ideal of relations between the generators $x_{i} y_{j}, 1 \leq i, j \leq 2$, of $S(V \oplus V)^{G}$ is a principal ideal generated by the relation $\left(x_{1} y_{1}\right)\left(x_{2} y_{2}\right)=\left(x_{1} y_{2}\right)\left(x_{2} y_{1}\right)$. It follows that $H\left(S(V \oplus V)^{G}, q\right)=\frac{1-q^{4}}{\left(1-q^{2}\right)^{4}}$. By (3) and (6) we obtain

$$
H\left(R^{G}, q\right)=D\left(\operatorname{ch}_{R}(q)\right)=D\left(\operatorname{ch}_{Z}(q)\right)=H\left(Z^{G}, q\right)=\frac{1}{1-q^{2}}+\frac{q^{2}\left(1+q^{2}\right)}{\left(1-q^{2}\right)^{4}} .
$$

Note that $R^{G}$ is not a finitely generated $K$-algebra. Indeed, $I$ is contained in $C^{2}$, the square of the commutator ideal of $K\langle V\rangle$, since $C^{2}$ is known to be the T-ideal of 2-variable identities of the subalgebra of upper triangular matrices in $K^{2 \times 2}$ (see 23]). Thus $K\langle V\rangle / C^{2}$ is a homomorphic image of $R$, and hence $\left(K\langle V\rangle / C^{2}\right)^{G}$ is a homomorphic image of $R^{G}$ (recall that $G$ is linearly reductive). Moreover, using 
an example from [22] it was pointed out in the proof of Theorem 3.1 in [1] that $\left(K\langle V\rangle / C^{2}\right)^{G}$ is not finitely generated, implying in turn that $R^{G}$ is not finitely generated.

Example 6.3. In some cases by a non-commutative variant of the Molien-Weyl theorem the operator $D$ from Section 3 can be evaluated using the Weyl integration formula and residue calculus. An instance where the Hilbert series of the subalgebra of invariants in a relatively free algebra was computed that way occurs in [10. Namely, take for $V$ the space of $2 \times 2$ matrices over $K$, on which $G=G L_{2}$ acts by conjugation, and let $I$ be the T-ideal in $K\langle V\rangle=K\left\langle x_{1}, x_{2}, x_{3}, x_{4}\right\rangle$ generated by $\left[\left[x_{1}, x_{2}\right], x_{2}\right]$. It was shown in Lemma 2.1 of [10] that

$$
H\left((K\langle V\rangle / I)^{G L_{2}}, t\right)=\frac{1+2 t^{3}+t^{4}}{(1-t)\left(1-t^{2}\right)} .
$$

In that case the algebra $K\langle V\rangle / I$ is Noetherian and hence $(K\langle V\rangle / I)^{G L_{2}}$ is finitely generated (see 11]). Explicit generators are also found in [10].

\section{TRANSFER PRINCIPLE}

In this section the characteristic of the base field $K$ can be positive, but we assume that $K$ is algebraically closed. Let $G$ be a linear algebraic group with coordinate ring $K[G]$, and let $H$ be a subgroup of $G$. Let $R$ be an associative $K$-algebra on which $G$ acts rationally via $K$-algebra automorphisms. Then $G \times H$ acts on $K[G] \otimes R$ via $K$-algebra automorphisms as follows:

$$
(g, h) \cdot \sum_{i} f_{i} \otimes r_{i}=\sum_{i} f_{i}\left(g^{-1} x h\right) \otimes g r_{i} \text { for } g \in G, h \in H, f_{i} \in K[G], r_{i} \in R,
$$

where we write $f_{i}\left(g^{-1} x h\right) \in K[G]$ for the function $G \rightarrow K, x \mapsto f_{i}\left(g^{-1} x h\right)$. Using the embeddings $G \rightarrow G \times H, g \mapsto(g, 1)$ and $H \rightarrow G \times H, h \mapsto(1, h)$, the $G \times H$ action on $K[G] \otimes R$ restricts to commuting actions of $G$ and $H$ on $K[G] \otimes R$. Recall that the subalgebra $(K[G] \otimes R)^{G}$ of $G$-invariants is stable under the $H$-action, and similarly, the subalgebra $(K[G] \otimes R)^{H}=K[G]^{H} \otimes R$ of $H$-invariants is $G$-stable. Clearly we have

$$
\left(K[G]^{H} \otimes R\right)^{G}=\left((K[G] \otimes R)^{H}\right)^{G}=(K[G] \otimes R)^{G \times H}=\left((K[G] \otimes R)^{G}\right)^{H} .
$$

Lemma 7.1. Denote by $\Phi:(K[G] \otimes R)^{G} \rightarrow R$ the restriction to $(K[G] \otimes R)^{G}$ of the linear map $K[G] \otimes R \rightarrow R$ given by $f \otimes r \mapsto f\left(1_{G}\right) r$. Then we have the following:

(i) $\Phi$ is an $H$-equivariant $K$-algebra isomorphism.

(ii) $\Phi$ restricts to a $K$-algebra isomorphism $\left(K[G]^{H} \otimes R\right)^{G} \rightarrow R^{H}$.

Proof. This is Theorem 9.1 in [19] (in [19] $R$ is assumed to be a commutative $K$ algebra, but commutativity is nowhere used in the proof, which works verbatim in the present generality).

Theorem 7.2. Let $R$ be a left noetherian finitely generated $K$-algebra satisfying a polynomial identity, and $G$ a linearly reductive linear algebraic group acting rationally on $R$ via $K$-algebra automorphisms. Suppose that $H$ is a subgroup of $G$ such that $K[G]^{H}$ is a finitely generated $K$-algebra (for example, $H$ can be a maximal unipotent subgroup of $G$ ). Then the subalgebra $R^{H}$ of $H$-invariants in $R$ is finitely generated and is left noetherian. 
Proof. Recall that the polynomial algebra $S[x]$ over a left noetherian ring $S$ is left noetherian (see for example [24]). An iterated use of this yields that since $K[G]^{H}$ is a finitely generated commutative $K$-algebra and $R$ is left noetherian, the algebra $K[G]^{H} \otimes R$ is left noetherian. As both $K[G]^{H}$ and $R$ are finitely generated $K$ algebras, the same holds for $K[G]^{H} \otimes R$. Moreover, $K[G]^{H} \otimes R$ is a PI-algebra, since it satisfies the same multilinear polynomial identities as $R$, and every PIalgebra over any field $K$ necessarily satisfies a multilinear polynomial identity. A theorem of Vonessen [31] asserts that the subalgebra of invariants of the linearly reductive group $G$ acting rationally on an affine left noetherian PI-algebra is finitely generated and left noetherian. By this theorem we conclude that $\left(K[G]^{H} \otimes R\right)^{G}$ is finitely generated and is left noetherian. The statement of the theorem now follows from the isomorphism $R^{H} \cong\left(K[G]^{H} \otimes R\right)^{G}$ in Lemma 7.1.

\section{REFERENCES}

[1] G. Almkvist, W. Dicks, E. Formanek, Hilbert series of fixed free algebras and noncommutative classical invariant theory, J. Algebra 93 (1985) 189-214.

[2] M. Atiyah, I. G. Macdonald, Introduction to Commutative Algebra (Addison-Wesley Publ., Reading, Mass, 1969).

[3] Yu. Bahturin, Identities of algebras with actions of Hopf algebras, Drensky, Vesselin S. (ed.) et al., Methods in Ring Theory, Proceedings of the Trento conference, Trento, Italy. New York, NY: Marcel Dekker. Lect. Notes Pure Appl. Math. 198, 1-36 (1998).

[4] A. Ya. Belov, Rationality of Hilbert series with respect to free algebras (Russian), Uspekhi Mat. Nauk 52(2) (1997) 153-154. Translation: Russian Math. Surveys 52 (2) (1997) 394-395.

[5] A. Berele, Applications of Belov's theorem to the cocharacter sequence of p.i. algebras, J. Algebra 298 (2006) 208-214.

[6] P. Borwein, M. Coons, Transcendence of power series for some number theoretic functions, Proc. Amer. Math. Soc. 137(4) (2009) 1303-1305.

[7] H. Derksen, G. Kemper, Computational Invariant Theory, Encyclopedia of Mathematical Sciences, 130 (Springer-Verlag, Berlin, 2002).

[8] I. Dolgachev, Lectures on Invariant Theory, London Math. Soc. Lecture Note Series 296, (Cambridge University Press, 2003).

[9] M. Domokos, Relatively free invariant algebras of finite reflection groups, Trans. Amer. Math. Soc. 348 (1996) 2217-2233.

[10] M. Domokos, Cayley-Hamilton theorem for $2 \times 2$ matrices over the Grassmann algebra, $J$. Pure Applied Algebra 133 (1998) 69-81.

[11] M. Domokos, V. Drensky, A Hilbert-Nagata theorem in noncommutative invariant theory, Trans. Amer. Math. Soc. 350(7) (1998) 2797-2811.

[12] V. Drensky, Commutative and noncommutative invariant theory, in Math. and Education in Math., Proc. of the 24-th Spring Conf. of the Union of Bulgar. Mathematicians, Svishtov, April 4-7, 1995, (Sofia, 1995), pp. 14-50.

[13] V. Drensky, E. Formanek, Polynomial Identity Rings, Advanced Courses in Mathematics, CRM Barcelona, (Birkhäuser Verlag, Basel, 2004).

[14] V. Drensky, C. K. Gupta, Constants of Weitzenböck derivations and invariants of unipotent transformations acting on relatively free algebras, J. Algebra 292 (2005) 393-428.

[15] M. S. Eryashkin, Invariants of the action of a semisimple Hopf algebra on PI-algebra (Russian), Izv. Vyssh. Uchebn. Zaved., Mat. 2016, No. 8 (2016), 21-34. Translation: Russ. Math. 60 (2016) 17-28.

[16] P. Fatou, Séries trigonométriques et séries de Taylor, Acta Math. 30(1) (1906) 335-400.

[17] E. Formanek, Noncommutative invariant theory, Contemp. Math. 43 (1985) 87-119.

[18] E. Formanek, P. Halpin, W.-C. W. Li, The Poincaré series of the ring of $2 \times 2$ generic matrices, J. Algebra 69 (1981) 105-112.

[19] F. D. Grosshans, Algebraic Homogeneous Spaces and Invariant Theory, Lecture Notes in Mathematics, 1673, (Springer-Verlag, Berlin, 1997).

[20] P. Grzeszczuk, M. Hryniewicka, Actions of pointed Hopf algebras with reduced PI invariants, Proc. Am. Math. Soc. 135 (2007) 2381-2389. 
[21] Dž. Hadžiev, Some questions in the theory of vector invariants (Russian), Mat. Sb. (NS) 72(114) (1967) 420-435. Translation: Math. USSR, Sb. 1 (1967) 383-396.

[22] V. K. Kharchenko, Noncommutative invariants of finite groups and Noetherian varieties, $J$. Pure Appl. Alg. 31 (1984) 83-90.

[23] Yu. N. Maltsev, A basis for the identities of the algebra of upper triangular matrices (Russian), Algebra i Logika 10 (1971) 393-400. Translation: Algebra Logic 10 (1971) 242-247.

[24] J. C. McConnell, J. C. Robson, Noncommutative Noetherian Rings (Wiley-Interscience, Chichester-New York, 1987).

[25] E. Miller, B. Sturmfels, Combinatorial Commutative Algebra, Graduate Texts in Mathematics 227, (Springer, New York, 2005).

[26] S. Mukai, An Introduction to Invariants and Moduli, Cambridge Studies in Advanced Mathematics 81, (Cambridge University Press, 2003).

[27] M. Nagata, On the 14-th problem of Hilbert, Amer. J. Math. 81 (1959) 766-772.

[28] M. B. Nathanson, Finitely generated and not finitely generated rings, arXiv:1606.00828v1 [math.AC].

[29] C. Procesi, Lie Groups (An Approach through Invariants and Representations) (Springer, New York, 2007).

[30] R. Steinberg, Nagata's example, in Algebraic groups and Lie groups, Austral. Math. Soc. Lect. Ser., 9, (Cambridge University Press, 1997), pp. 375-384.

[31] N. Vonessen, Actions of Linearly Reductive Groups on Affine PI-Algebras, Mem. Amer. Math. Soc. 81(414) (1989).

MTA Alfréd Rényi Institute of Mathematics, ReÁltanoda utca 13-15, 1053 Budapest, HUNGARY

E-mail address: domokos.matyas@renyi.mta.hu

Institute of Mathematics and Informatics, Bulgarian Academy of Sciences, Acad. G. Bonchev Str., Block 8, 1113 Sofia, Bulgaria

E-mail address: drensky@math.bas.bg 